\title{
VERA status and results
}

\section{H. Kobayashi ${ }^{1,2}$ and the VERA collaboration}

${ }^{1}$ Department of Astronomy, Graduate School of Science, The University of Tokyo, 7-3-1 Hongo, Bunkyo-ku, Tokyo 113-0033, Japan email: hideyuki.kobayashi@nao.ac.jp

${ }^{2}$ National Astronomical Observatory of Japan, 2-21-1 Osawa, Mitaka, Tokyo 181-8588, Japan

\begin{abstract}
VERA is a Very Long Baseline Interferometry (VLBI) array for astrometry, composed of four $20 \mathrm{~m}$ radio telescopes. They are located over a range of around $2300 \mathrm{~km}$ in Japan. VERA consists of a two-beam system equipped with $2,6.7,8,22$, and $43 \mathrm{GHz}$ receivers. The two-beam system is used for phase referencing of the VLBI observations, to compensate for atmospheric-turbulence effects between two nearby objects. It has achieved measurements of annual parallaxes within $5 \mathrm{kpc}$ with $10 \%$ accuracy. Observed sources are water, $\mathrm{SiO}$, and methanol masers, which are found in molecular gas around star-forming regions and evolved stars. We have carried out a large program of astrometry to reveal the Galaxy's structure and velocity field. VERA has already measured trigonometric parallaxes of more than 30 sources and observed around a hundred sources using the two-beam astrometry technique. Maser sources are associated with high-mass star-forming regions, which are thought to trace the arm structure of the Galaxy. Using annual parallax and proper-motion measurements, their structure will be shown without kinematic distance assumptions. Some sources exhibit large differences between trigonometric-parallax measurements and kinematic distances. We present the status of the VERA project as well as recent results.
\end{abstract}

Keywords. masers, instrumentation: interferometers, techniques: interferometric, astrometry, Galaxy: structure 